\title{
CENTRAL ELEMENTS FOR QUANTUM AFFINE ALGEBRAS AND AFFINE MACDONALD'S OPERATORS
}

\author{
PAVEl I. Etingof
}

\section{Introduction}

A theorem of Harish-Chandra says that the center of the universal enveloping algebra $U(\mathfrak{g})$ of a complex simple Lie algebra $\mathfrak{g}$ is isomorphic to the Grothendieck algebra of the category of finite dimensional representations of $\mathfrak{g}$. However, this theorem does not furnish a natural identification of these two algebras.

The situation improves when we pass from Lie algebras to quantum groups. The structure of the center of the quantum group $U_{q}(\mathfrak{g})$ was described by V. Drinfeld [Dr1] and N. Reshetikhin [R]. It turns out that there exists a natural isomorphism from the representation ring to the center, constructed with the help of the quasi-triangular structure of $U_{q}(\mathfrak{g})$ (Theorem 1), which allows us to describe the central elements and the Harish-Chandra homomorphism explicitly.

The Drinfeld-Reshetikhin construction generalizes to an arbitrary symmetrizable Kac-Moody algebra $\mathfrak{g}$. For infinite dimensional $\mathfrak{g}$ the obtained central elements will be in a completion of $U_{q}(\mathfrak{g})$. Such central elements, for $q=1$, have already been constructed by Kac $[\mathrm{K}]$, with the help of the contravariant form. As in the finite dimensional case, for general $q$, one can obtain a more explicit description of these elements.

We describe this generalization in the case of quantum affine algebras. In this case, central elements correspond to Weyl group invariant thetafunctions from [Lo] (Theorem 2).

In $[\mathrm{EK} 1, \mathrm{EK} 2]$ it was shown that the action of central elements of $U_{q}\left(s l_{n}\right)$ on certain intertwining operators produces Macdonald's difference operators. This can be regarded as the statement that Macdonald's operators

Received March 27, 1995.

Partially supported by an NSF postdoctoral fellowship. 
are "radial parts" of "quantum Laplace operators", and Macdonald's polynomials (or, more generally, eigenfunctions of Macdonald's operators) are the corresponding $q$-spherical functions (Theorems 3, 4).

We apply the same construction in the quantum affine case and obtain affine analogues of Macdonald's difference operators. They are not difference operators in the usual sense but rather infinite sums of difference operators. It is not easy to compute them explicitly, even for the Lie algebra $\widehat{s l_{2}}$, but they commute, preserve the space of symmetric theta-functions, and are triangular with respect to the standard ordering of affine weights. This shows that they have a unique, up to factors, common eigenbasis in the space of symmetric theta-functions, which is exactly the basis of affine Macdonald's polynomials defined in [EK3] (Theorems 5, 6). We hope that these operators and their eigenfunctions (affine Macdonald functions) will be understood better in the near future.

\section{The center of $U_{q}(\mathfrak{g})$, where $\mathfrak{g}$ is a simple Lie algebra}

1.1. Let $\mathfrak{g}$ be a simple Lie algebra over $\mathbb{C}$ of rank $r$. Let $\mathfrak{h}$ be a Cartan subalgebra in $\mathfrak{g}$. Let $W$ be the Weyl group. We fix a Weyl group invariant inner product $\langle$,$\rangle on \mathfrak{h}^{*}$ and $\mathfrak{h}$ by setting $\langle\alpha, \alpha\rangle=2$ for short roots. Fix a polarization of $\mathfrak{g}$. Let $\rho \in \mathfrak{h}^{*}$ be the half-sum of positive roots. Let $Q$ be the root lattice of $\mathfrak{g}, Q^{+}$be the semigroup with 0 spanned by the positive roots. Let $\alpha_{1}, \ldots, \alpha_{r}$ be the simple roots. Let $A=\left(a_{i j}\right), a_{i j}=2\left\langle\alpha_{i}, \alpha_{j}\right\rangle /\left\langle\alpha_{i}, \alpha_{i}\right\rangle$ be the Cartan matrix of $\mathfrak{g}$. Let $P$ be the weight lattice of $\mathfrak{g}, P^{+}$be the set of dominant integral weights, and $\omega_{1}, \ldots, \omega_{r}$ be the fundamental weights. Let $N$ be the order of $P / Q$. Note that for any $\lambda, \mu \in P, N\langle\lambda, \mu\rangle \in \mathbb{Z}$.

1.2. Let $\hat{q}$ be a formal variable. Let $q=\hat{q}^{N}$. For any $a \in \frac{1}{N} \mathbb{Z}$ we define $q^{a}:=\hat{q}^{N a}$.

Let $U_{q}(\mathfrak{g})$ be the quantum group corresponding to $\mathfrak{g}([\mathrm{Dr} 1, \mathrm{~J} 1])$. It is a Hopf algebra freely generated over the field $F=\mathbb{C}(\hat{q})$ by the elements $E_{i}$, $F_{i}, 1 \leq i \leq r$, and $K_{\beta}, \beta \in P$, modulo the relations:

$$
\begin{gathered}
K_{\beta} K_{\gamma}=K_{\beta+\gamma}, K_{0}=1, K_{\beta} E_{i} K_{-\beta}=q^{\left\langle\beta, \alpha_{i}\right\rangle} E_{i}, \\
K_{\beta} F_{i} K_{-\beta}=q^{-\left\langle\beta, \alpha_{i}\right\rangle} F_{i},\left[E_{i}, F_{j}\right]=\delta_{i j} \frac{k_{i}-k_{i}^{-1}}{q_{i}-q_{i}^{-1}}, q_{i}=q^{\frac{1}{2}\left\langle\alpha_{i}, \alpha_{i}\right\rangle}, k_{i}=K_{\alpha_{i}}, \\
\\
\sum_{l=0}^{1-a_{i j}}(-1)^{l}\left[\begin{array}{c}
1-a_{i j} \\
l
\end{array}\right]_{q_{i}} E_{i}^{1-a_{i j}-l} E_{j} E_{i}^{l}=0, i \neq j,
\end{gathered}
$$




$$
\sum_{l=0}^{1-a_{i j}}(-1)^{l}\left[\begin{array}{c}
1-a_{i j} \\
l
\end{array}\right]_{q_{i}} F_{i}^{1-a_{i j}-l} F_{j} F_{i}^{l}=0, i \neq j
$$

where

$$
\left[\begin{array}{c}
m \\
l
\end{array}\right]_{q}:=\prod_{j=0}^{l-1} \frac{[m-j]_{q}}{[l-j]_{q}}, \quad[m]_{q}:=\frac{q^{m}-q^{-m}}{q-q^{-1}} .
$$

The comultiplication is defined by

$\Delta\left(E_{i}\right)=E_{i} \otimes k_{i}+1 \otimes E_{i}, \quad \Delta\left(F_{i}\right)=F_{i} \otimes 1+k_{i}^{-1} \otimes F_{i}, \quad \Delta\left(K_{\beta}\right)=K_{\beta} \otimes K_{\beta}$.

The antipode is given by

$$
S\left(E_{i}\right)=-E_{i} k_{i}^{-1}, \quad S\left(F_{i}\right)=-k_{i} F_{i}, \quad S\left(K_{\beta}\right)=K_{-\beta} .
$$

The counit $\varepsilon$ annihilates $E_{i}$ and $F_{i}$ and maps $K_{\beta}$ to the identity.

1.3. Let $K$ be an extension of $F$. We call any character $\theta: P \rightarrow K^{*}$ a $K$-weight for $U_{q}(\mathfrak{g})$. We say that a representation $V$ of $U_{q}(\mathfrak{g}) \otimes K$ is with highest weight $\theta$ if it is generated by a vector $v$ such that $E_{i} v=0,1 \leq i \leq r$, and $K_{\beta} v=\theta(\beta) v, \beta \in P$. The product of two characters is just the pointwise product.

Note that any character $\theta: P \rightarrow K^{*}$ extends to a ring homomorphism $\mathbb{C}[P] \rightarrow K$, acting by $\theta\left(\sum c_{\lambda} e^{\lambda}\right)=\sum c_{\lambda} \theta(\lambda)$, where $e^{\lambda}$ is a basis of $\mathbb{C}[P]$. We denote this homomorphism also by $\theta$.

In particular, for $\mu \in P$, we can define $e^{\mu}: P \rightarrow F^{*}$ by $e^{\mu}(\lambda)=q^{\langle\mu, \lambda\rangle}$.

1.4. Let $\mathcal{O}_{f}$ be the category of finite dimensional representations $V$ of $U_{q}(\mathfrak{g})$ such that $V=\oplus_{\lambda \in P} V\left[e^{\lambda}\right],\left.K_{\beta}\right|_{V\left[e^{\lambda}\right]}=q^{\langle\beta, \lambda\rangle} I d$. For $\mu \in P^{+}$, let $V_{\mu} \in \mathcal{O}_{f}$ be the irreducible representation with highest weight $e^{\mu}$.

1.5. Let $U_{q}\left(\mathfrak{n}^{ \pm}\right)$be the subalgebras in $U_{q}(\mathfrak{g})$ generated by $\left\{E_{i}\right\},\left\{F_{i}\right\}$, respectively. Let $\left\{a_{i}, i \geq 0\right\}$ be a homogeneous basis of $U_{q}\left(\mathfrak{n}^{+}\right)\left(a_{0}=1\right)$, and let $\left\{a_{i}^{*}\right\}$ denote the dual basis to $\left\{a_{i}\right\}$ with respect to the Drinfeld pairing between $U_{q}\left(\mathfrak{n}^{+}\right)$and $U_{q}\left(\mathfrak{n}^{-}\right)$[Dr1].

1.6. Let $V \in \mathcal{O}_{f}$. Define an element $C_{V} \in U_{q}(\mathfrak{g})$ by

$$
C_{V}:=\left.\sum_{i, j, \lambda} q^{2\langle\lambda, \rho\rangle} \operatorname{Tr}\right|_{V\left[e^{\lambda}\right]}\left(a_{i} a_{j}^{*}\right) a_{i}^{*} K_{2\left(\lambda-\beta_{j}\right)} a_{j}
$$


where $\beta_{i}$ is the weight of $a_{i}$, i.e., $K_{\gamma} a_{i} K_{-\gamma}=q^{\left\langle\gamma, \beta_{i}\right\rangle} a_{i}, \gamma \in P$. Clearly, the sum in (1) is finite; almost all terms vanish.

Remark. The element $C_{V}$ can be shortly written in the form

$$
\left.\operatorname{Tr}\right|_{V}\left(R_{21} R\left(1 \otimes K_{2 \rho}\right)\right)
$$

where $R$ is the universal $R$-matrix for $U_{q}(\mathfrak{g})$ [Dr1], and $R_{21}$ is the result of permuting the two components of $R$.

\section{7 .}

Theorem 1. [Dr1], [R]

(i) $C_{V}$ belongs to the center $\mathcal{Z}$ of $U_{q}(\mathfrak{g})$.

(ii) (quantum Harish-Chandra's theorem) Let Rep $\mathfrak{g}$ be the Grothendieck ring of the category $\mathcal{O}_{f}$ as a tensor category. Then the map $V \rightarrow C_{V}$ is a ring isomorphism $F \otimes_{\mathbb{Z}} \operatorname{Rep} \mathfrak{g} \rightarrow \mathcal{Z}$. Thus, $\left\{C_{V_{\mu}}, \mu \in P^{+}\right\}$is a basis of $\mathcal{Z}$, and $\mathcal{Z}=F\left[C_{V_{\omega_{1}}}, \ldots, C_{V_{\omega_{r}}}\right]$.

(iii) (formula for the Harish-Chandra homomorphism) Let $K$ be an extension of $F$ and $M$ be a highest weight representation of $U_{q}(\mathfrak{g}) \otimes K$ with highest weight $\sigma: P \rightarrow K^{*}$. Then $\left.C_{V}\right|_{M}=\theta\left(\chi_{V}\right)$, where $\theta=\sigma^{2} e^{2 \rho}$, and $\chi_{V} \in \mathbb{C}[P]$ is the character of $V$.

\section{Generalization to the affine case}

2.1. Let $\tilde{\mathfrak{g}}=\mathfrak{g} \otimes \mathbb{C}\left[t, t^{-1}\right] \oplus \mathbb{C} c \oplus \mathbb{C} d$ be the affine Lie algebra corresponding to $\mathfrak{g}$. Let $\tilde{\mathfrak{h}}=\mathfrak{h} \oplus \mathbb{C} c \oplus \mathbb{C} d$ be the Cartan subalgebra in $\mathfrak{g}$. Then $\tilde{\mathfrak{h}}^{*}=$ $\mathfrak{h}^{*} \oplus \mathbb{C} \varepsilon \oplus \mathbb{C} \delta$, so that $\varepsilon(c)=\delta(d)=1$, and $\left.\delta\right|_{\mathfrak{h} \oplus \mathbb{C} c}=\left.\varepsilon\right|_{\mathfrak{h} \oplus \mathbb{C} d}=0$. We can extend the form $\langle$,$\rangle to a nondegenerate form on \tilde{\mathfrak{h}}^{*}$ and $\tilde{\mathfrak{h}}$ by setting $\langle\varepsilon, \delta\rangle=1$, and $\langle\varepsilon, \varepsilon\rangle=\langle\delta, \delta\rangle=\left\langle\varepsilon, \mathfrak{h}^{*}\right\rangle=\left\langle\delta, \mathfrak{h}^{*}\right\rangle=0$. Define $\alpha_{0}=\delta-\tilde{\alpha}$, where $\tilde{\alpha}$ is the maximal root of $\mathfrak{g}$. Then the simple roots of $\tilde{\mathfrak{g}}$ are $\alpha_{0}, \ldots, \alpha_{r}$. The affine Weyl group $\hat{W}$ is generated by the finite Weyl group $W$ and an additional reflection $s_{0}$ acting by $s_{0} \lambda=\lambda-2 \frac{\left.<\lambda, \alpha_{0}\right\rangle}{\left.<\alpha_{0}, \alpha_{0}\right\rangle} \alpha_{0}$. It acts in a natural way on $\tilde{\mathfrak{h}}^{*}$. This action preserves $\langle$,$\rangle .$

Let the weight $\hat{\rho} \in \tilde{\mathfrak{h}}^{*}$ be defined by the condition $\left\langle\hat{\rho}, \alpha_{i}\right\rangle=\frac{1}{2}\left\langle\alpha_{i}, \alpha_{i}\right\rangle$ for all simple roots $\alpha_{i}, i=0, \ldots, r$, and $\langle\hat{\rho}, \varepsilon\rangle=0$. Then $\hat{\rho}=\rho+g \varepsilon$, where $g$ is the dual Coxeter number of $\mathfrak{g}$.

Let $\hat{\omega}_{j} \in \tilde{\mathfrak{h}}^{*}, 0 \leq j \leq r$, be the fundamental weights of $\tilde{\mathfrak{g}}$, defined by $\left\langle\hat{\omega}_{j}, \alpha_{i}\right\rangle=\frac{\delta_{i j}}{2}\left\langle\alpha_{i}, \alpha_{i}\right\rangle$, and $\left\langle\hat{\omega}_{j}, \varepsilon\right\rangle=0$. Define $\tilde{P}$ to be the set of all weights of the form $\lambda=m \delta+\sum_{j=0}^{r} m_{j} \hat{\omega}_{j}, m, m_{j} \in \mathbb{Z}$. Let $\tilde{P}^{+}$be the set of 
dominant integral weights, i.e., weights of the form $\lambda=m \delta+\sum_{j=0}^{r} m_{j} \hat{\omega}_{j}$, $m \in \mathbb{Z}, m_{j} \in \mathbb{Z}_{+}$. Let $\tilde{Q}$ be the root lattice of $\tilde{\mathfrak{g}}$, and let $\tilde{Q}^{+}$be the semigroup with 0 spanned by the positive roots.

2.2. Let $U_{q}(\tilde{\mathfrak{g}})$ be the quantum affine algebra corresponding to $\tilde{\mathfrak{g}}([\operatorname{Dr} 1])$. It is defined as follows. Its generators (over $F$ ) are $E_{i}, F_{i}, i=0, \ldots, r, K_{\beta}$, $\beta \in \tilde{P}$, which satisfy exactly the same relations as in Section 1 , with the only difference that $i, j$ vary from 0 to $r$, and $\beta$ varies over $\tilde{P}$. Let $U_{q}(\hat{\mathfrak{g}})$ be the subalgebra in $U_{q}(\tilde{\mathfrak{g}})$ generated by $E_{i}, F_{i}, K_{\beta}, \beta \in \tilde{P},\langle\beta, \delta\rangle=0$.

Let $\left\{e^{\mu}, \mu \in \tilde{P}\right\}$ be the natural basis of $\mathbb{C}[\tilde{P}]$. Let $F[[\tilde{P}]]_{T}$ denote the ring of formal series $\sum_{m=0}^{\infty} c_{m} e^{\lambda_{m}}, c_{m} \in F, \lambda_{m} \in \tilde{P}$, such that $\left\langle\lambda_{m}, \hat{\rho}\right\rangle \rightarrow-\infty$, $m \rightarrow \infty$, and the order of the pole of the function $c_{m}$ at $\hat{q}=0$ and $\hat{q}=\infty$ is bounded from above as a function of $m$. We call such formal series tempered (this is why we use the subscript $\mathrm{T}$ in the notation). Let $A$ be the intersection of all the translates of $F[[\tilde{P}]]_{T}$ under the action of the affine Weyl group. (It is easy to see that $\hat{W}$ does not preserve $F[[\tilde{P}]]_{T}$.) For $\phi=\sum c_{m} e^{\lambda_{m}} \in A$, we formally set $\xi(\phi)=\sum c_{m} q^{\left\langle\lambda_{m}, \hat{\rho}\right\rangle} K_{\lambda_{m}}$.

Let $U_{q}\left(\hat{\mathfrak{n}}^{ \pm}\right)$be the subalgebras in $U_{q}(\tilde{\mathfrak{g}})$ generated by $\left\{E_{i}\right\},\left\{F_{i}\right\}$, respectively. Let $\left\{a_{i}, i \geq 0\right\}$ be a homogeneous basis of $U_{q}\left(\hat{\mathfrak{n}}^{+}\right)\left(a_{0}=1\right)$, and let $\left\{a_{i}^{*}\right\}$ denote the dual basis to $\left\{a_{i}\right\}$ with respect to the Drinfeld pairing between $U_{q}\left(\hat{\mathfrak{n}}^{+}\right)$and $U_{q}\left(\hat{\mathfrak{n}}^{-}\right)$.

Let $\widehat{U_{q}(\tilde{\mathfrak{g}})}$ denote the completion of $U_{q}(\tilde{\mathfrak{g}})$ consisting of all formal series of the form $\sum_{i, j} a_{i}^{*} \xi\left(\phi_{i j}\right) a_{j}$, where $\phi_{i j} \in A$, and the number of distinct weights of terms in the sum is finite (so that $\widehat{U_{q}(\tilde{\mathfrak{g}})}$ is a $\tilde{P}$-graded algebra). The algebra structure on $\widehat{U_{q}(\tilde{\mathfrak{g}})}$ is obvious.

2.3. Let $K$ be an extension of $F$. We call any character $\theta: \tilde{P} \rightarrow K^{*}$ a $K$-weight for $U_{q}(\tilde{\mathfrak{g}})$. We say that a representation $V$ of $U_{q}(\mathfrak{g}) \otimes K$ is with highest weight $\theta$ if it is generated by a vector $v$ such that $E_{i} v=0,0 \leq i \leq r$, and $K_{\beta} v=\theta(\beta) v, \beta \in \tilde{P}$. In particular, for $\mu \in \tilde{P}$ we can define $e^{\mu}: \tilde{\tilde{P}} \rightarrow F^{*}$ by $e^{\mu}(\lambda)=q^{\langle\mu, \lambda\rangle}$.

Now assume that $K=L(s)$, where $s$ is a transcendental element over a field $L \supset F$, and $\theta\left(\alpha_{j}\right) \in L\left[s^{-1}\right], \theta(\delta) \in s^{-1} L\left[s^{-1}\right]$. We call such a weight admissible. Then the map $\theta$ extends to an algebra homomorphism $A \rightarrow L((s))$. Indeed, if $a=\sum c_{m} e^{\lambda_{m}} \in A$ then $\left\langle\lambda_{m}, \varepsilon\right\rangle \rightarrow-\infty, m \rightarrow \infty$, so $\theta(a)$ converges in $L((s))$. Note that the set of admissible weights is invariant under shifting by roots of $\tilde{\mathfrak{g}}$.

Moreover, if $\theta=e^{\mu}, \mu \in \tilde{P}$, and $\langle\mu, \delta\rangle>0$, then $\theta$ extends to a ring homomorphism $A \rightarrow F_{-}, F_{-}=\mathbb{C}\left(\left(\hat{q}^{-1}\right)\right)$. It follows from the fact that for 
such $\mu$, we have $\left\langle\mu, \lambda_{m}\right\rangle \rightarrow-\infty, m \rightarrow \infty$. Similarly, if $\langle\mu, \delta\rangle<0$ then $\theta$ extends to a ring homomorphism $A \rightarrow F_{+}, F_{+}=\mathbb{C}((\hat{q}))$. For convenience we denote by $\tilde{P}( \pm)$ the subsets of $\tilde{P}$ consisting of all $\mu \in \tilde{P}$ such that $\langle\mu, \delta\rangle \in \mathbb{N}^{ \pm}$.

2.4. Let $M$ be a module over $U_{q}(\tilde{\mathfrak{g}}) \otimes K$, where $K$ is an extension of $F$. For any weight $\theta: \tilde{P} \rightarrow K^{*}$ we denote by $M[\theta]$ the space of all vectors of weight $\theta$ in $M$. We say that $M$ has weight decomposition if $M=\oplus_{\theta} M_{\theta}$. We say that $M$ is smooth if it has a weight decomposition, and $U_{q}\left(\hat{\mathfrak{n}}^{+}\right) v$ is a finite dimensional space for any $v \in M$. Let $\mathcal{S}_{K}$ be the category of smooth modules. Let $\mathcal{S}_{L}^{a}$ be the full subcategory of $\mathcal{S}_{L(s)}$ consisting of all smooth modules having only admissible weights, and $\mathcal{S}_{ \pm}$be the full subcategory of $\mathcal{S}_{F}$ consisting of modules having only weights of the form $e^{\mu}, \mu+\hat{\rho} \in \tilde{P}( \pm)$. Then, as follows from Section 2.3, for any $M \in S_{L}^{a}, M \otimes L((s))$ naturally extends to a module over $\widehat{U_{q}(\tilde{\mathfrak{g}})} \otimes L((s))$, and for any $M \in \mathcal{S}_{ \pm}, M \otimes F_{\mp}$ naturally extends to a module over $\widehat{U_{q}(\tilde{\mathfrak{g}})} \otimes F_{\mp}$. The action of $\xi(A)$ in these extensions are defined by $\left.\xi(\phi)\right|_{M[\theta]}=\left(\theta e^{\hat{\rho}}\right)(\phi) \mathrm{Id}$.

2.5. We would like to construct some central elements in $\widehat{U_{q}(\tilde{\mathfrak{g}})}$. One of these elements is obvious - it is $K_{\delta}$. The rest of the central elements can be constructed analogously to Drinfeld's method.

Let $U_{q}(\tilde{\mathfrak{g}})_{2}$ be the subalgebra in $U_{q}(\tilde{\mathfrak{g}})$ generated by $U_{q}(\hat{\mathfrak{g}})$ and $K_{\varepsilon}^{2}$. It is clear that $U_{q}(\tilde{\mathfrak{g}})_{2}$ is a Hopf subalgebra, and $U_{q}(\tilde{\mathfrak{g}})=U_{q}(\tilde{\mathfrak{g}})_{2} \oplus K_{\varepsilon} U_{q}(\tilde{\mathfrak{g}})_{2}$. Let $\tilde{P}_{2}$ be the sublattice of $\tilde{P}$ spanned by $\hat{\omega}_{i}$ and $2 \delta$, and let $\tilde{P}_{1 / 2}$ be the lattice of $\tilde{P}$ spanned by $\hat{\omega}_{i}$ and $\frac{1}{2} \delta$. If $\lambda \in P_{1 / 2}$, we define the character $e^{\lambda}: P_{2} \rightarrow F^{*}$ by the usual formula $e^{\lambda}(\beta)=q^{\langle\lambda, \beta\rangle}$.

Let $\mathcal{O}_{i}$ be the category of integrable representations $V$ of $U_{q}(\tilde{\mathfrak{g}})_{2}$ such that $V=\oplus_{\lambda \in \tilde{P}_{1 / 2}} V\left[e^{\lambda}\right], \operatorname{dim} V\left[e^{\lambda}\right]<\infty$, and $\left.K_{\beta}\right|_{V\left[e^{\lambda}\right]}=q^{\langle\beta, \lambda\rangle} I d, \beta \in \tilde{P}_{2}$. (Integrability means that the restriction of $V$ to any $U_{q}\left(s l_{2}\right)$-subalgebra corresponding to a simple root is a direct sum of finite dimensional modules). This category is semisimple $([\mathrm{L}])$ : any object is a (possibly infinite) direct sum of irreducible objects.

For $\mu \in P_{1 / 2}$, let $V_{\mu} \in \mathcal{O}_{i}$ be the irreducible representation of $U_{q}(\tilde{\mathfrak{g}})_{2}$ with highest weight $e^{\mu}$. Any simple object in $\mathcal{O}_{i}$ is isomorphic to $V_{\mu}$ for a unique $\mu$. Characters of representations $V \in \mathcal{O}_{i}$ are infinite linear combinations of $e^{\mu}, \mu \in \tilde{P}_{1 / 2}$.

Clearly, $\mathcal{O}_{i}$ is closed with respect to the tensor product. 
2.6. Let $V \in \mathcal{O}_{i}$. Define an element $C_{V} \in U_{q}(\tilde{\mathfrak{g}})$ by

$$
C_{V}:=\left.\sum_{i, j, \lambda} q^{2\langle\lambda, \hat{\rho}\rangle} \operatorname{Tr}\right|_{V\left[e^{\lambda}\right]}\left(a_{i} a_{j}^{*}\right) a_{i}^{*} K_{2\left(\lambda-\beta_{j}\right)} a_{j}
$$

where $\beta_{i}$ is the weight of $a_{i}$. Clearly, the sum in (2) is a well defined element of $\widehat{U_{q}(\tilde{\mathfrak{g}})}$. For example, if $V_{n}$ is the 1-dimensional $U_{q}(\tilde{\mathfrak{g}})_{2}$-module in which $U_{q}(\hat{\mathfrak{g}})$ acts trivially and $K_{\varepsilon}^{2}$ acts by multiplication by $q^{n}$ then $C_{V_{n}}=q^{g n} K_{\delta}^{n}$.

Remarks. 1. The element $C_{V}$ can be shortly written in the form

$$
\left.\operatorname{Tr}\right|_{V}\left(R_{21} R\left(1 \otimes K_{2 \hat{\rho}}\right)\right)
$$

where $R$ is the universal R-matrix for $U_{q}(\tilde{\mathfrak{g}})$ [Dr1], and $R_{21}$ is the result of permutation of the two components of $R$.

2. The reason we need to introduce the algebra $U_{q}(\tilde{\mathfrak{g}})_{2}$ is the following. If we only consider elements $C_{V}$ with $V$ being an integrable $U_{q}(\tilde{\mathfrak{g}})$-module, like we did in the finite dimensional case, then the algebra generated by all such elements will not contain $K_{\delta}$ (although it will contain its square).

2.7. The following theorem is the affine analogue of Drinfeld's theorem.

\section{Theorem 2.}

(i) $C_{V}$ belongs to the center $\tilde{\mathcal{Z}}$ of $\widehat{U_{q}(\tilde{\mathfrak{g}})}$.

(ii) Let Rep $\tilde{\mathfrak{g}}$ be the Grothendieck ring of the category $\mathcal{O}_{i}$ as a tensor category. Then the map $V \rightarrow C_{V}$ is a ring isomorphism $F \otimes_{\mathbb{Z}}$ $\operatorname{Rep} \tilde{\mathfrak{g}} \rightarrow \tilde{\mathcal{Z}}$. Thus, $\left\{C_{V_{\mu}}, \mu \in \tilde{P}^{+}\right\}$is a topological basis of $\tilde{\mathcal{Z}}$, and $\tilde{\mathcal{Z}}=F\left[C_{V_{\hat{\omega}_{0}}}, \ldots, C_{V_{\hat{\omega}_{r}}}\right]\left(\left(K_{\delta}\right)\right)_{T}$ (here, as before, the subscript $T$ means the tempered series, i.e., such that the order of poles of its coefficients at $\hat{q}=0$ and $\hat{q}=\infty$ are bounded from above).

(iii) Let $L$ be an extension of $F, K=L(s)$ be the field of rational functions of $s$. Let $M$ be a highest weight representation of $U_{q}(\tilde{\mathfrak{g}}) \otimes K$ with an admissible highest weight $\sigma: \tilde{P} \rightarrow K^{*}$. Then $M \otimes L((s))$ extends to a representation of $\widehat{U_{q}(\tilde{\mathfrak{g}})} \otimes L((s))$, and $C_{V}$ acts there as $\theta\left(\chi_{V}\right) I d$, where $\chi_{V}$ is the character of $V$, and $\theta=\sigma^{2} e^{2 \hat{\rho}}$.

(iv) Let $\nu \in \tilde{P}$ and $\langle\nu, \delta\rangle+g \in \mp \mathbb{N}$. Let $M$ be any representation of $U_{q}(\tilde{\mathfrak{g}})$ with highest weight $\nu$. Then $M \otimes F_{ \pm}$extends to a representation of $\widehat{U_{q}(\tilde{\mathfrak{g}})} \otimes F_{ \pm}$in a natural way, and $C_{V}$ acts there as $e^{2(\nu+\hat{\rho})}\left(\chi_{V}\right) I d$, where $\chi_{V}$ is the character of $V$. 


\section{8 .}

Remarks. 1. The statement about topological basis in (ii) means that any element $z \in \tilde{\mathcal{Z}}$ can be uniquely represented as $\sum_{n} b_{n} C_{V_{\mu_{n}}}, b_{n} \in F$, and $\left\langle\mu_{n}, \hat{\rho}\right\rangle \rightarrow-\infty$ as $n \rightarrow \infty$.

2. We formulate (iv) separately from (iii) because weights in $\tilde{P}$ are not admissible.

3. This theorem is easily generalized to the case of $U_{q}(\mathfrak{a})$ - quantization of an arbitrary symmetrizable Kac-Moody algebra $\mathfrak{a}$. The changes which need to be made are obvious, and we do not discuss them here.

4. In the classical case $q=1$, Kac $[\mathrm{K}]$ constructed central elements in a completion of $U(\mathfrak{a})$, where $\mathfrak{a}$ an arbitrary symmetrizable Kac-Moody algebra. Kac's construction assigns such a central element to any function on the Tits cone in the set of weights, in such a way that the this assignment inverts the Harish-Chandra homomorphism. Kac's construction easily generalizes to quantum groups, and the elements $C_{V}$ are examples of central elements one can obtain using this construction. But in the quantum case

we also have nice explicit formulas like (1), (2), which are absent in the classical case.

\section{9 .}

Proof of Theorem 2 (analogous to the proof of Theorem 1).

(i) Let $V_{1}, V_{2}$ be highest weight representations of $U_{q}(\tilde{\mathfrak{g}})$, and let

$$
X: V_{1} \otimes V_{2} \rightarrow V_{1} \otimes V_{2}
$$

be an intertwining operator. The trace $\left.\operatorname{Tr}\right|_{V_{2}}\left(X\left(1 \otimes K_{2 \hat{\rho}}\right)\right)$ is assumed to make sense. Then $X$ defines an intertwining operator $V_{1} \rightarrow V_{1}$, i.e., it commutes with the action of $U_{q}(\tilde{\mathfrak{g}})$. Therefore, (i) follows from Remark 2.6 and the fact that $R_{21} R$ commutes with $\Delta(a), a \in U_{q}(\tilde{\mathfrak{g}})$.

(iii, iv) When we apply $C_{V}$ to the highest weight vector $v \in M$, all terms in (2) with $i, j>0$ vanish, and what remains is

$$
C_{V} v=\sum_{\lambda} q^{\langle 2 \lambda, \hat{\rho}\rangle} \operatorname{dim} V\left[e^{\lambda}\right] K_{2 \lambda} v=\theta\left(\chi_{V}\right) v .
$$

Since $M$ is generated by $v$, we get (iii) and (iv).

(ii) The fact that the assignment $V \rightarrow C_{V}$ is a ring homomorphism follows from the fusion property of the $R$-matrix. Moreover, Kac $[\mathrm{K}]$ 
showed that a central element $C \in \widehat{U_{q}(\tilde{\mathfrak{g}})}$ of the form $\sum a_{i}^{*} \xi\left(\phi_{i j}\right) a_{j}$ is completely determined by $\phi_{00}$ (strictly speaking, Kac's paper treats the case $q=1$, but the results we need are obviously valid in the quantum case). Thus, the map $V \rightarrow C_{V}$ is injective. To show that it is surjective, let us recall $[\mathrm{K}]$ that for any $\lambda \in \tilde{P}$, and any character $\sigma: \tilde{P} \rightarrow F^{*}$ such that $\sigma(\tilde{Q}) \subset\{ \pm 1\}\left(\sigma e^{\lambda}\right)\left(\phi_{00}\right)=$ $\left(\sigma e^{s_{\alpha}(\lambda)}\right)\left(\phi_{00}\right)$ when $\langle\lambda+\hat{\rho}, \alpha\rangle=\frac{n}{2}\langle\alpha, \alpha\rangle$, where $n \in \mathbb{N}, \alpha$ is a positive root of $\tilde{\mathfrak{g}}$, and $s_{\alpha}$ is the Weyl reflection with respect to $\alpha$ (the Kac-Kazhdan condition). Indeed, the Verma module with highest weight $\sigma e^{\lambda}$ contains the Verma module with highest weight $\sigma e^{s_{\alpha}(\lambda+\hat{\rho})-\hat{\rho}}$ when the Kac-Kazhdan condition is satisfied (for $\sigma=1$ this is obvious, and in general it follows from the fact that $\sigma$ is a highest weight of a 1-dimensional representation of $\left.U_{q}(\tilde{\mathfrak{g}})\right)$, and $C_{V}$ acts by $\left(\sigma e^{\lambda+\hat{\rho}}\right)\left(\phi_{00}\right) I d$ on the Verma module with highest weight $\sigma e^{\lambda}$. Therefore, $\phi_{00} \in A^{\hat{W}}$, where $A^{\hat{W}}$ is the set of $\hat{W}$-invariant elements in $A$. Moreover, by varying the choice of $\sigma$ we see that if a monomial $e^{\lambda}$ occurs in $\phi_{00}$ with a nontrivial coefficient, then the coefficients to all $\omega_{i}$ in $\lambda$ are even.

But the algebra $A^{\hat{W}}$ is nothing else but the completed algebra of invariant theta functions associated to the affine root system of $\tilde{\mathfrak{g}}$. By a theorem of Looijenga ([Lo]), this algebra is topologically spanned over $F$ by the characters of integrable representations, so, taking into account the evenness property, we get (ii).

Remarks. 1. As we have seen, the construction of the center for $U_{q}(\hat{\mathfrak{g}})$ given above works at any non-critical value of the central charge: $K_{\delta} \neq q^{-g}$. At the critical value $q^{-g}$, the structure of the center is different. In fact, in this case the center is much bigger - it contains an algebra of polynomials of infinitely many variables. However, even in this case the above construction (formula (2)) allows to obtain a central element in a completion of $U_{q}(\hat{\mathfrak{g}})$ if the module $V$ is taken not from the category $\mathcal{O}_{i}$ but from the category of finite dimensional $U_{q}(\hat{\mathfrak{g}})$-modules. This construction was discovered in [RS]; see also [DE].

2. The constructions of central elements for $U_{q}(\mathfrak{g}), U_{q}(\hat{\mathfrak{g}})$ by formulas (1), (2), as well as the construction of central elements of $U_{q}(\hat{\mathfrak{g}})$ at the critical level given in [RS, DE], mentioned in the previous remark, are special cases of the general construction of categorical trace defined by J. Bernstein [B], as follows. 
Let $A, B$ be categories, $F: A \rightarrow B$ be a functor, and $F^{*},{ }^{*} F: B \rightarrow A$ be right and left adjoint functors to $F$. Let $\varepsilon: F^{*} \rightarrow^{*} F$ be a morphism of functors. Let $X$ be an object in $A$ and $a: F X \rightarrow F X$ be an endomorphism. Consider the morphism $\operatorname{tr}(a): X \rightarrow X$ defined as the composition of morphisms $\operatorname{tr}(a)=i_{X} \circ{ }^{*} F(a) \circ \varepsilon_{F X} \circ j_{X}$, where $i_{X}: F^{*} F X \rightarrow X$, $j_{X}: X \rightarrow^{*} F F X$ are the adjunction morphisms. This defines the linear operator of categorical trace $t r: \operatorname{End}(F) \rightarrow \operatorname{End}\left(I d_{A}\right)$, where $I d_{A}: A \rightarrow A$ is the identity functor.

Let $U$ be an associative algebra over a $\operatorname{ring} \mathcal{F}$, and $A$ be a full subcategory of the category of $U$-modules. Then the center $Z(U)$ of $U$ naturally maps to the ring $\operatorname{End}(F)$, where $F$ is any functor on $A$. In particular, if $F$ is the identity functor, this map is often an embedding. Therefore $Z(U)$ can often be identified with a subring of the ring of endomorphisms of the identity functor on the category of representations of $U$.

It is shown in $[\mathrm{B}]$ how to use the trace construction to produce many central elements of $U$ when we have only one fixed central element $C \in$ $Z(U)$. Indeed, let $F: A \rightarrow A$ be any functor satisfying the above conditions. Then we can consider $\operatorname{tr} F(C) \in \operatorname{End}\left(I d_{A}\right)$, and if we are lucky, so that this element is in the image of the center, then we get a new central element $C_{F} \in Z(U)$. Let us demonstrate how this works for quantum groups.

If $U$ is a quasi-triangular Hopf algebra (e.g., $\left.U_{q}(\mathfrak{g}), U_{q}(\hat{\mathfrak{g}})\right)$ then one can define an element $u=m\left((S \otimes 1)\left(R_{21}\right)\right)$ in a completion of this algebra, where $m$ is the multiplication map, $S$ is the antipode, and $R_{21}$ is the universal $R$ matrix with permuted components. Conjugation by $u$ is $S^{2}$. This element was defined by Drinfeld [Dr2]. It is easy to show that the element $Z=$ $u S(u)$ is central. Moreover, in the cases of $U_{q}(\mathfrak{g}), U_{q}(\hat{\mathfrak{g}})$ it is possible to find a central element $C$ such that $\Delta(C)=(C \otimes C)\left(R_{21} R\right)$, and $C^{2}=Z^{-1}$ (it is obtained by formal extraction of square root from $Z^{-1}$; Hopf algebras where $C$ exists are called ribbon Hopf algebras, [RT]). Now, if $V$ is an irreducible, integrable highest weight module, and $F=F_{V}$ is the functor of tensoring of $U$-modules with $V$ (on the right). Then ${ }^{*} F, F^{*}$ are the functors of tensoring with ${ }^{*} V, V^{*}$, respectively, and $u: V^{*} \rightarrow^{*} V$ defines an isomorphism $\varepsilon: F^{*} \rightarrow^{*} F$. It is easy to see that $C_{V}=\frac{1}{\left.C\right|_{V}} C^{-1} C_{F}$, where $C_{F}$ is the categorical trace of $C$, and $\left.C\right|_{V}$ is the eigenvalue of $C$ in $V$. This explains formulas (1), (2).

\section{Central elements and Macdonald operators}

3.1. Macdonald operators acting on the space of rational functions of $n$ 
variables over $\mathbb{C}(q, t)$ are defined as follows:

$$
M^{r}=t^{r(r-n)} \sum_{i_{1}<i_{2}<\ldots<i_{r}}\left(\prod_{\substack{j \notin\left\{i_{1} \ldots i_{r}\right\} \\ l=1 \ldots r}} \frac{t^{2} x_{i_{l}}-x_{j}}{x_{i_{l}}-x_{j}}\right) T_{i_{1}} \ldots T_{i_{r}}
$$

where $\left(T_{i} f\right)\left(x_{1}, \ldots, x_{n}\right)=f\left(x_{1}, \ldots, q^{2} x_{i}, \ldots, x_{n}\right), t$ is a formal variable, and $r=1, \ldots, n-1$.

Macdonald proved $[\mathrm{M}]$ that these operators are pairwise commutative: $\left[M^{r}, M^{s}\right]=0,1 \leq r, s \leq n-1$.

In [EK1],[EK2] it was shown how to obtain Macdonald operators from central elements of the quantum group $U_{q}(\mathfrak{g})$, where $\mathfrak{g}=\mathfrak{s l}_{n}$. This was done as follows.

3.2. Recall that fundamental representations of $U_{q}(\mathfrak{g})$ are $q$-deformed exterior powers $\left(\Lambda^{r} F^{n}\right)^{*}, r=1, \ldots, n-1$. Consider the elements

$$
C_{r}:=C_{\left(\Lambda^{r} F^{n}\right)^{*}} \in U_{q}(\mathfrak{g}),
$$

defined by (1), where $\rho=\left(\frac{n-1}{2}, \frac{n-3}{2}, \ldots, \frac{-n+1}{2}\right)$. These elements are central and freely generate the center of $U_{q}(\mathfrak{g})$, according to Theorem 1 .

3.3. Let $\hat{t}$ be a formal variable such that $\hat{t}^{2}=t$. Let $K$ be an extension of $F(\hat{t})$, and let $\theta: P \rightarrow K^{*}$ be a weight. Let $M_{\theta}$ denote the Verma module over $U_{q}(\mathfrak{g})$ with highest weight $\theta$, and let

$$
U=\left\{p \in K\left[x_{1}^{ \pm 1}, \ldots, x_{n}^{ \pm 1}\right], \operatorname{deg} p=0\right\}
$$

with the action of $U_{q}(\mathfrak{g}) \otimes K$ given by

$$
\begin{gathered}
E_{i} \mapsto x_{i} D_{i+1}, F_{i} \mapsto x_{i+1} D_{i}, \\
K_{\beta} f\left(x_{1}, \ldots, x_{n}\right)=f\left(q^{\beta_{1}} x_{1}, \ldots, q^{\beta_{n}} x_{n}\right), \beta=\left(\beta_{1}, \ldots, \beta_{n}\right) \in P \\
\left(D_{i} f\right)\left(x_{1}, \ldots, x_{n}\right) \\
=\frac{t q^{-1} f\left(x_{1}, \ldots, q x_{i}, \ldots, x_{n}\right)-t^{-1} q f\left(x_{1}, \ldots, q^{-1} x_{i}, \ldots, x_{n}\right)}{\left(q-q^{-1}\right) x_{i}}
\end{gathered}
$$

The set of weights of $U$ is the root lattice $Q$, and every weight subspace is one-dimensional: $U[\mu]=K x_{1}^{\mu_{1}} \ldots x_{n}^{\mu_{n}}, \mu=\left(\mu_{1}, \ldots, \mu_{n}\right) \in Q$. We fix a vector $u \in U[0]$. 


\section{4 .}

Lemma 1. If $M_{\theta}$ is irreducible, then there exists a unique intertwiner

$$
\Phi_{\theta}: M_{\theta} \rightarrow M_{\theta} \hat{\otimes} U
$$

normalized by the condition $\Phi_{\theta} v=v \otimes u+$ lower order terms, where $v$ is the highest weight vector of $M_{\theta}$, and $\hat{\otimes}$ denotes the completed tensor product with respect to the principal grading in $M_{\theta}$.

Proof is based on the general fact: if a Verma module $M_{\theta}$ is irreducible and $U$ is any $U_{q}(\mathfrak{g}) \otimes K$-module then the space of intertwiners $M_{\theta} \rightarrow M_{\theta} \otimes U$ is in one-to-one correspondence with the zero-weight subspace $U[0]$, i.e the space of $K_{\beta}$-invariant vectors in $U$ (for all $\beta \in P$ ).

Remark. The module $M_{\theta}$ over $U_{q}(\mathfrak{g})$ is reducible if and only if the quantum Kac-Kazhdan condition is satisfied: $\theta(2 \alpha)=q^{n\langle\alpha, \alpha\rangle}$ for some positive root $\alpha$ of $\mathfrak{g}$, and some integer $n>0$. This follows from the formula for the determinant of the Shapovalov form, see [DCK].

3.5. Let $Y=\operatorname{Hom}\left(P, K^{*}\right)$. Define a completion $K[[Y]]$ of the group algebra $K[Y]$ of $Y$. By definition, $K[[Y]]$ is spanned by the subspaces $K[[Y]]_{\sigma}, \sigma \in Y$, where $K[[Y]]_{\sigma}$ consists of all, possibly infinite, formal series of the form $\sum_{\beta \in Q^{+}} c_{\beta} \sigma e^{-\beta}$, where $c_{\beta} \in K$.

Let $M$ be a highest weight $U_{q}(\mathfrak{g}) \otimes K$-module. For any $\Phi: M \rightarrow M \hat{\otimes} U$ denote by $\Phi[\theta]$ the diagonal block of $\Phi$ of weight $\theta$ :

$$
\Phi[\theta]: M[\theta] \rightarrow M[\theta] \otimes U[0] .
$$

Let $K(Y)$ be the quotient field of $K[Y]$, and let $X: M \otimes K(Y) \rightarrow M \otimes K(Y)$ be the linear operator defined by $X v=\theta v, v \in M[\theta]$.

Let $\tau_{0}: P \rightarrow K^{*}$ be defined by $\tau_{0}(\lambda)=\hat{t}^{2\langle\lambda, \rho\rangle}$, and let $\tau=\tau_{0} e^{-\rho}$. Let $\chi=\theta \tau$.

Set

$$
\Psi_{\theta}=\left.\operatorname{Tr}\right|_{M_{\chi} \otimes K(Y)}\left(\Phi_{\chi} X\right):=\sum_{\beta \in Q^{+}} \operatorname{Tr}\left(\Phi_{\chi}\left[\chi e^{-\beta}\right]\right) \chi e^{-\beta}
$$

This is an element of $K[[Y]] \otimes U[0]$. The construction of $\Psi_{\theta}$ is valid for a generic $\theta \in Y$, i.e., such that the corresponding Verma module $M_{\chi}$ is irreducible.

Since the space $U[0]$ is one-dimensional, we can identify it with $K$ by sending $u$ to 1 and regard $\Psi_{\theta}$ as a series with scalar coefficients, i.e., as an element of $K[[Y]]$. 
3.6. Now we explain a connection between Macdonald difference operators and the series $\Psi_{\theta}$.

Definition. A standard difference operator is any Laurent polynomial in the operators $T_{i}$ whose coefficients are rational functions of

$$
y_{1}=x_{2} / x_{1}, \ldots, y_{n-1}=x_{n} / x_{n-1}(\operatorname{over} F(\hat{t}))
$$

which are regular at $y_{1}=\ldots=y_{n-1}=0$.

It is clear that the algebra of standard difference operators can be embedded in the algebra $K\left[\left[y_{1}, \ldots, y_{n-1}\right]\right]\left[T_{1}, \ldots, T_{n}\right]$ (by taking the Taylor expansion of the coefficients). It therefore follows that any standard difference operator naturally acts on the space $K[[Y]]_{\sigma}, \sigma \in Y$. This action is given by $T_{j} \theta=\theta\left(\omega_{j}-\omega_{j-1}\right)^{2} \theta$ (by convention $\omega_{0}=0$ ), and $y_{j} \theta=\theta e^{-\alpha_{j}}$. There is a natural power series topology on $K[[Y]]_{\sigma}$, and the action of any standard difference operator is continuous in this topology.

\section{7.}

Theorem 3. ([EK2])

(i) For every $r$ between 1 and $n-1$ there exists a unique standard difference operator $\tilde{M}^{r}$ defined over $F(t)$, such that

$$
\left.\operatorname{Tr}\right|_{M_{\chi} \otimes K(Y)}\left(\Phi_{\chi} C_{r} X\right)=\left.\tilde{M}^{r} \operatorname{Tr}\right|_{M_{\chi} \otimes K(Y)}\left(\Phi_{\chi} X\right)
$$

for all $\chi$ not satisfying the Kac-Kazhdan condition.

(ii) $\left[\tilde{M}^{r}, \tilde{M}^{s}\right]=0,1 \leq r, s \leq n-1$.

(iii) Introduce the series

$$
\varphi=\tau \prod_{i=1}^{\infty} \prod_{\alpha \in R^{+}} \frac{1-q^{2 i} e^{-\alpha}}{1-q^{2(i-1)} t^{2} e^{-\alpha}} \in \operatorname{AutK}[[Y]]
$$

where $R^{+}$is the set of positive roots of $\mathfrak{g}$, and $\tau$ denotes the operator of multiplication by $\tau$ in $K[[Y]]$. Then $\frac{1}{\varphi} \tilde{M}^{r}(\varphi f)=M^{r} f$ for any $f \in K[[Y]]$, where $M^{r}$ are the Macdonald operators defined by (3). Thus, for any $\theta \in Y$ for which $\Psi_{\chi}$ is defined, the series $\psi_{\theta}:=\Psi_{\chi} / \varphi$ is a common eigenvector of the Macdonald operators in $K[[Y]]: M^{r} \psi_{\theta}=P_{r}(\theta) \psi_{\theta}$, where $P_{r}(\theta)=\left(\theta \tau_{0}\right)^{2}\left(\chi_{r}\right)$, and $\chi_{r}$ is the character of $\left(\Lambda^{r} F^{n}\right)^{*}$. 
(iv) Let $\sigma \in Y$ be such that the operator $\Phi_{\chi}$ exists for any $\theta=\sigma e^{-\beta}$, $\beta \in Q^{+}$. Then the vectors $\psi_{\sigma e^{-\beta}}, \beta \in Q^{+}$, form a topological basis of $K[[Y]]_{\sigma}$ (with respect to the usual power series topology). Thus, $M^{r}$ are simultaneously diagonalizable in $K[[Y]]_{\sigma}$. Generically, each of them has a simple spectrum in $K[[Y]]_{\sigma}$.

(v) Let $\sigma \in Y$ be a weight such that the weights $\sigma^{w}:=w\left(\sigma \tau_{0}\right) \tau_{0}^{-1}$, $w \in S_{n}$, are all distinct, and $\Phi_{\sigma^{w} \tau}$ exists for each $w$. Then the system of difference equations $M^{r} \psi=P_{r}(\sigma) \psi, r \in 1, \ldots, n-1$, has $n$ ! linearly independent solutions in $K[[Y]]$. They are $\psi_{\sigma^{w}}, w \in S_{n}$.

3.8. In particular, the construction of functions $\psi_{\theta}$ can be specialized to the case when $\theta=e^{\lambda}, \lambda$ is a weight in $P^{+}$. Lemma 1 implies that the operator $\Phi_{\chi}$ always exists in this case, and we have

Theorem 4. If $\lambda \in P^{+}$then the series $\psi_{e^{\lambda}}$ belongs to $\mathbb{C}(q, t)[P]$ and coincides with the Macdonald polynomial $P_{\lambda}(x ; q, t)$ (see [EK2]).

\section{Affine Macdonald operators}

In this section we will define an affine analogue of Macdonald operators using the analogy with Section 3. We consider the case $\mathfrak{g}=\operatorname{sl}(n)$.

4.1. Let $p: U_{q}(\hat{\mathfrak{g}}) \rightarrow U_{q}(\mathfrak{g})$ be the Jimbo homomorphism [J2]. Let $U(1)=$ $p^{*}(U)$.

Let $\theta: \tilde{P} \rightarrow K^{*}$ be a weight, and let $M_{\theta}$ be the Verma module over $U_{q}(\tilde{\mathfrak{g}}) \otimes K$ with highest weight $\theta$.

\section{2.}

Lemma 2. If $M_{\theta}$ is irreducible, there exists a unique $U_{q}(\hat{\mathfrak{g}})$-intertwiner

$$
\Phi_{\theta}: M_{\theta} \rightarrow M_{\theta} \hat{\otimes} U(1),
$$

normalized by the condition $\Phi_{\theta} v=v \otimes u+$ lower order terms, where $v$ is the highest weight vector of $M_{\theta}$, and $\hat{\otimes}$ denotes the completed tensor product with respect to the principal grading in $M_{\theta}$.

Proof is analogous to the finite dimensional case.

Operators of this form for quantum affine algebras were introduced by Frenkel and Reshetikhin [FR].

Remark. The module $M_{\theta}$ over $U_{q}(\hat{\mathfrak{g}})$ is reducible if and only if the KacKazhdan condition is satisfied: $\theta(2 \alpha)=q^{n\langle\alpha, \alpha\rangle}$ for some root $\alpha>0$ of $\tilde{\mathfrak{g}}$ and some integer $n>0$. 
4.3. Let $\tilde{Y}=\operatorname{Hom}\left(\tilde{P}, K^{*}\right)$. Define a completion $K[[\tilde{Y}]]$ of the group algebra $K[\tilde{Y}]$ of $\tilde{Y}$. By definition, $K[[\tilde{Y}]]$ is spanned by the subspaces $K[[\tilde{Y}]]_{\sigma}, \sigma \in \tilde{Y}$, where $K[[\tilde{Y}]]_{\sigma}$ consists of all, possibly infinite, formal series of the form $\sum_{\beta \in \tilde{Q}^{+}} c_{\beta} \sigma e^{-\beta}$, where $c_{\beta} \in K$.

Let $M$ be a highest weight $U_{q}(\hat{\mathfrak{g}}) \otimes K$-module. For any $\Phi: M \rightarrow M \hat{\otimes} U$ denote by $\Phi[\theta]$ the diagonal block of $\Phi$ of weight $\theta$ :

$$
\Phi[\theta]: M[\theta] \rightarrow M[\theta] \otimes U[0]
$$

Let $K(\tilde{Y})$ be the quotient field of $K[\tilde{Y}]$, and let $X: M \otimes K(\tilde{Y}) \rightarrow M \otimes K(\tilde{Y})$ be the linear operator defined by $X v=\theta v, v \in M[\theta]$.

Let $\tau_{0}: \tilde{P} \rightarrow K^{*}$ be defined by $\tau_{0}(\lambda)=\hat{t}^{2\langle\lambda, \hat{\rho}\rangle}$, and let $\tau=\tau_{0} e^{-\hat{\rho}}$.

Set

$$
\Psi_{\theta}=\left.\operatorname{Tr}\right|_{M_{\chi} \otimes K(\tilde{Y})}\left(\Phi_{\chi} X\right):=\sum_{\beta \in \tilde{Q}^{+}} \operatorname{Tr}\left(\Phi_{\chi}\left[\chi e^{-\beta}\right]\right) \chi e^{-\beta}
$$

This is an element of $K[[\tilde{Y}]] \otimes U[0]$. The construction of $\Psi_{\theta}$ is valid for a generic $\theta \in \tilde{Y}$, i.e., such that the corresponding Verma module $M_{\chi}$ is irreducible.

Since the space $U[0]$ is one-dimensional, we can identify it with $K$ by sending $u$ to 1 and regard $\Psi_{\theta}$ as a series with scalar coefficients, i.e., as an element of $K[[\tilde{Y}]]$.

\section{4 .}

Definition. An affine difference operator is a formal expression of the form $M=\sum a_{m} T_{\nu_{m}}, \nu_{m} \in \tilde{P}$, such that for any $w \in \hat{W}\left\langle w \nu_{m}, \hat{\rho}\right\rangle \rightarrow-\infty$, $m \rightarrow \infty$, and $a_{m} \in F(t)[[\tilde{Y}]]_{1}$, where 1 denotes the identity character $\tilde{P} \rightarrow \mathbb{C}^{*}$.

Affine difference operators form an algebra, in which multiplication is defined by $T_{\nu} \theta=\theta(\nu)^{2} \theta T_{\nu}$. This formula also gives rise to a natural action of the algebra of affine difference operators in $K[[\tilde{Y}]]_{\sigma} \otimes L((s))$ for any admissible $\sigma \in \tilde{Y}$. Also, if the series $M$ is tempered, in the sense of Section 2, the action of $M$ is defined in $G_{ \pm}[[\tilde{Y}]]_{\sigma}, \sigma \in \tilde{P}(\mp), G_{ \pm}=\mathbb{C}(\hat{t})\left(\left(\hat{q}^{ \pm 1}\right)\right)$. These actions are continuous in the standard power series topology. We will further assume that all highest weights we consider are either admissible or from $\tilde{P}( \pm)$. 
4.5. The proof of the following is analogous to the proof of Theorem 3:

\section{Theorem 5.}

(i) For every $r$ between 0 and $n-1$ there exists a unique affine difference operator $\tilde{M}^{\hat{\omega}_{r}}$ such that

$$
\operatorname{Tr}\left(\Phi_{\chi} C_{V_{\hat{\omega}_{r}}} X\right)=\tilde{M}^{\hat{\omega}_{r}} \operatorname{Tr}\left(\Phi_{\chi} X\right)
$$

for all $\chi$ not satisfying the Kac-Kazhdan condition.

(ii) $\left[\tilde{M}^{\hat{\omega}_{r}}, \tilde{M}^{\hat{\omega}_{s}}\right]=0,0 \leq r, s \leq n-1$.

Uniqueness: an affine difference operator is uniquely determined by its action in $K[[\tilde{Y}]]_{\sigma}$ for generic $\sigma$, and this action is defined uniquely by (12). Indeed, suppose we have an expression $\sum a_{m} T_{\nu_{m}}$ which acts by 0 on $K[[\tilde{Y}]]_{\sigma}$ for a generic admissible weight $\sigma$. In particular, applying it to $\sigma$, we get $\sum a_{m} \sigma\left(\nu_{m}\right)^{2}=0$. This identity for generic admissible $\sigma$ implies immediately that $a_{m}=0$ for all $m$.

4.6. Introduce the series

$$
\varphi=\tau \prod_{i=1}^{\infty} \prod_{\alpha \in \tilde{R}^{+}} \frac{1-q^{2 i} e^{-\alpha}}{1-q^{2(i-1)} t^{2} e^{-\alpha}} \in F(t)[[\tilde{Y}]]_{1},
$$

where $\tilde{R}^{+}$is the set of positive roots of $\tilde{\mathfrak{g}}$.

Introduce the affine difference operators $M^{\hat{\omega}_{r}}$ defined by

$$
M^{\hat{\omega}_{r}} f=\frac{1}{\varphi} \tilde{M}^{\hat{\omega}_{r}}(\varphi f)
$$

for any $f \in K[[\tilde{Y}]]$. These operators are pairwise commutative.

Definition. We call the operators $M^{\hat{\omega}_{r}}$ affine Macdonald operators.

4.7. For any $\theta$ for which $\Psi_{\chi}$ is defined consider the series $\psi_{\theta}:=\Psi_{\theta} / \varphi$.

\section{Corollary.}

(i) $\psi_{\theta}$ is a common eigenvector of the Macdonald operators in $K[[\tilde{Y}]]_{\theta}$ : $M^{\hat{\omega}_{r}} \psi_{\theta}=\tilde{P}_{r}(\theta) \psi_{\theta}$, where $\tilde{P}_{r}(\theta)=\left(\theta \tau_{0}\right)^{2}\left(\chi_{\hat{\omega}_{r}}\right)$, and $\chi_{\hat{\omega}_{r}}$ is the character of the $r$ th fundamental representation.

(ii) Let $\sigma \in \tilde{Y}$ be such that the operator $\Phi_{\chi}$ exists for any $\theta=\sigma e^{-\beta}$, $\beta \in \tilde{Q}^{+}$. Then the vectors $\psi_{\sigma e^{-\beta}}, \beta \in \tilde{Q}^{+}$, form a topological basis of $K[[\tilde{Y}]]_{\sigma}$ (with respect to the usual power series topology). 
Thus, $M^{\hat{\omega}_{r}}$ are simultaneously diagonalizable in $K[[\tilde{Y}]]_{\sigma}$. Generically, each of them has a simple spectrum in $K[[\tilde{Y}]]_{\sigma}$.

(iii) Let $\sigma \in \tilde{Y}$ be a weight such that the weights $\sigma^{w}:=w\left(\sigma \tau_{0}\right) \tau_{0}^{-1}$, $w \in \hat{W}$, are all distinct, and $\Phi_{\sigma^{w} \tau}$ exists for each $w$. Then the space of solutions of the system of difference equations $M^{\hat{\omega}_{r}} \psi=\tilde{P}_{r}(\sigma) \psi$ is topologically spanned by the linearly independent solutions $\psi_{\sigma^{w}}$, $w \in \hat{W}$.

The proof is analogous to the finite dimensional case.

4.8. In particular, the construction of functions $\psi_{\theta}$ can be specialized to the case when $\theta=e^{\lambda}, \lambda$ is a weight in $P^{+}$. Then the weight $\chi$ is admissible $\left(K=F(s), s=\hat{t}^{-1}\right)$, and Lemma 2 implies that the operator $\Phi_{\chi}$ exists. In this case, we have

Theorem 6. [EK3] If $\lambda \in P^{+}$then the function $\psi_{e^{\lambda}}$ belongs to $\mathbb{C}(\hat{q}, \hat{t}) \otimes_{F}$ $A^{\hat{W}}$ and coincides with the affine Macdonald polynomial $\hat{J}_{\lambda}$ defined in [EK3].

(In fact, it is obvious that the coefficients of $\hat{J}_{\lambda}$ are in $\left.\mathbb{C}(q, t)\right)$. As shown in [EK3], affine Macdonald polynomials are a basis of $\mathbb{C}(\hat{q}, \hat{t}) \otimes_{F} A^{\hat{W}}$, triangular with respect to the basis of characters $\chi_{\lambda}$. Therefore, we find:

Corollary. The operators $M^{\hat{\omega}_{r}}$ are $\hat{W}$-invariant.

This follows from the fact that an affine difference operator is uniquely defined by its action on symmetric theta-functions.

Thus, analogously to the finite dimensional case, affine Macdonald operators act on the space of symmetric theta-functions and are triangular with respect to the standard ordering of dominant weights. Affine Macdonald polynomials can be defined as their common eigenbasis.

In particular, it follows from this corollary that the coefficients to all $T_{\nu}$ in the affine Macdonald operators are of the form $\sum c_{m} e^{-\alpha_{m}}$, where $\left\langle w \alpha_{m}, \hat{\rho}\right\rangle \rightarrow+\infty, m \rightarrow \infty$, for all $w \in \hat{W}$. However, these coefficients need not be Weyl group invariant.

\section{Acknowledgements}

I would like to thank H. Garland who explained to me the idea of a (conjectured) different construction of affine Macdonald operators. I am grateful to P. Polo for explaining to me the connection of paper [B] with construction of the center of quantum groups. I am grateful to J. Bernstein, I. Frenkel, A. Kirillov Jr., and D. Kazhdan for discussions. 


\section{References}

[B] J. Bernstein, Trace in categories, Operator algebras, unitary representations, enveloping algebras, and invariant theory, 1990, pp. 417-424.

[Dr1] V. G. Drinfeld, Quantum groups, Proc. Int. Congr. Math., Berkeley, 1986, 798820.

[Dr2] - On almost cocommutative Hopf algebras, Len. Math. J. 1 (1990), no. 2, $321-342$.

[DCK] C. De Concini and V. Kac, Quantum groups at roots of 1, Operator algebras, unitary representations, enveloping algebras, and invariant theory, 1990, pp. 471508.

[DE] J. Ding and P. Etingof, Center of a quantum affine algebra at the critical level, Math. Res. Let. 1 (1994), no. 4, 469-480.

[EK1] P. I. Etingof and A. A. Kirillov, Jr, A unified representation-theoretic approach to special functions, hep-th 9312101, Functional Anal. and its Applic. 28 (1994), no. 1.

[EK2] Macdonald's polynomials and representations of quantum groups, Math. Res. Let. 1 (1994), no. 3, 279-296.

[EK3] - On the affine analogue of Jack and Macdonald polynomials, to appear in Duke Math. J., hep-th 9403168 (1994).

[FR] I. B. Frenkel and N. Yu. Reshetikhin, Quantum affine algebras and holonomic difference equations, Comm. Math. Phys. 146 (1992), 1-60.

[J1] M. A. Jimbo, A q-difference analogue of $U \mathfrak{g}$ and the Yang-Baxter equation, Lett. Math. Phys. 10 (1985), 62-69.

[J2] A q-analogue of $U(\mathfrak{g l}(N+1))$, Hecke algebra and the Yang-Baxter equation, Lett. Math. Phys. 11 (1986), 247-252.

[K] V. Kac, Laplace operators of infinite dimensional Lie algebras and theta functions, Proc. Nat. Acad. Sci. USA 81 (1984), 645-647.

[L] G. Lusztig, Introduction to quantum groups, Birkhauser, Boston, 1994.

[Lo] E. Looijenga, Root systems and elliptic curves, Inv. Math. 33 (1976), 17-32.

[M] I. G. Macdonald, A new class of symmetric functions, Publ. I.R.M.A. Strasbourg, 372/S-20, Actes 20 Séminaire Lotharingien (1988), 131-171.

[R] N. Yu. Reshetikhin, Quasitriangle Hopf algebras and invariants of tangles, Len. Math. J. 1 (1990), 491-513.

[RS] N. Yu. Reshetikhin and M. A. Semenov-Tian-Shansky, Central extensions of quantum current groups, Lett. Math. Phys. 19 (1990), 133-142.

[RT] N. Yu. Reshetikhin and V. G. Turaev, Ribbon graphs and their invariants derived from quantum groups, Invent. Math. 103 (1991), 547-597.

Department of Mathematics, Harvard Univerity, Cambridge, M A 02138

E-mail address: etingof@math.harvard.edu 